\title{
Intravenous Iron in Oncology
}

\author{
Michael Auerbach, MD, a,b and Harold Ballard, MD, Baltimore, Maryland, and New York, New York
}

\section{Key Words}

Anemia, intravenous iron, erythropoiesis-stimulatory agents, chemotherapy-induced anemia

\begin{abstract}
Intravenous iron (IV Fe) as an adjunct to therapy with erythropoiesisstimulatory agents (ESAs) is standard care in dialysis-associated anemia, adding huge increments in hemoglobin and hematopoietic responses and decreased transfusions without significant toxicity. Cost savings, decreased exposure to ESAs, and decreased times to reach target hemoglobins are realized. Although similar benefits have been seen in all studies performed in patients with chemotherapy-induced anemia ( $\mathrm{CIA})$, experts are reluctant to incorporate routine use of IV Fe into treatment, largely because of misinterpretation and misunderstanding of the clinical nature of adverse events reportedly associated with its administration. IV Fe is therefore underused in oncology patients with anemia. Published experience with more than 1000 patients in clinical trials involving the use of IV Fe suggests minimal toxicity and substantial benefit are experienced when high molecular weight iron dextran is avoided. This article presents evidence recommending routine incorporation of IV Fe into treatment for CIA. (JNCCN 2008;6:585-592)
\end{abstract}

From a Georgetown University, Washington, DC, bPrivate Practice, Baltimore, Maryland, and 'New York University School of Medicine, Harborview VA Medical Center, New York, New York.

Submitted December 21, 2007; accepted for publication March 31, 2008.

The authors have no financial interest, arrangement, or affiliation with the manufacturers of any products discussed in the article or their competitors.

Correspondence: Michael Auerbach, MD, Private Practice, 9110 Philadelphia Road \#314, Baltimore, MD 21237.

E-mail: mauerbachmd@aol.com

\section{EDITORS}

Kerrin G. Robinson, MA, Medical/Scientific Editor, Journal of the National Comprehensive Cancer Network

Disclosure: Kerrin G. Robinson, MA, has disclosed no relevant financial relationships.

\section{CME Author}

Désirée Lie, MD, MSEd, Clinical Professor, Family Medicine, University of California, Orange; Director, Division of Faculty Development, UCI Medical Center, Orange, California

Disclosure: Désirée Lie, MD, MSEd, has disclosed no relevant financial relationships.

\section{Medscape: Continuing Medical Education Online}

Medscape, LLC is pleased to provide online continuing medical education (CME) for this journal article, allowing clinicians the opportunity to earn CME credit. Medscape, LLC is accredited by the Accreditation Council for Continuing Medical Education (ACCME) to provide CME for physicians. Medscape, LLC designates this educational activity for a maximum of 0.75 AMA PRA Category 1 Credits $^{\mathrm{TM}}$. Physicians should only claim credit commensurate with the extent of their participation in the activity. All other clinicians completing this activity will be issued a certificate of participation. To participate in this journal CME activity: (1) review the learning objectives and author disclosures; (2) study the education content; (3) take the post-test and/or complete the evaluation at www.medscape.com; (4) view/print certificate.

\section{Learning Objectives}

Upon completion of this activity, participants will be able to:

- Identify erythropoiesis stimulatory agents (ESAs) approved and licensed for use in the United States for oncology patients

- Describe the most likely mechanisms accounting for anemia among patients with cancer

- Define characteristics of functional iron deficiency in oncology patients

- Provide the most likely explanation for a suboptimal response to ESAs in oncology patients

- Describe the recommendations of the authors for the use of intravenous iron in oncology patients

In August 2007, the Committee on Medicare and Medicaid Services issued a Decision Memo restricting the use of erythropoiesis-stimulatory agents (ESAs) in patients with cancer- and chemotherapy-related anemia. The use of ESAs has been proscribed in all patients with cancer-related anemia not undergoing chemotherapy and patients with chemotherapy-induced anemia (CIA) with hemoglobin levels greater than $10 \mathrm{~g} / \mathrm{dL}$. The National Institute for Health and Clinical Excellence in the United Kingdom recommends ESAs not be used unless clinically significant anemia is present and transfusions are unavailable. ${ }^{1}$ These decisions were based on 3 randomized, placebo-controlled studies showing negative cancer outcomes in patients receiving ESAs compared with placebo..$^{2-4}$ In 2 of these studies, nonanemic oncology patients were treated to supratherapeutic 
Auerbach and Ballard

hemoglobin levels. ${ }^{2,3}$ All 3 studies had imbalances in pretreatment prognostic indicators favoring the placebo group.

In the United States and worldwide, $\$ 4$ and $\$ 6$ billion, respectively, are spent per year on ESAs. Oncologists spend 3 times as much per patient for half the transfusion decrement seen in dialysis patients. ${ }^{5}$ Although virtually $100 \%$ of patients undergoing dialysis receive intravenous iron (IV Fe) as an adjunct to ESA therapy, fewer than $10 \%$ of oncology patients are estimated to receive IV Fe when appropriate. ${ }^{6}$ In dialysis-associated anemia, the target hemoglobin level remains 12 to $13 \mathrm{~g} / \mathrm{dL}$. No restriction of ESA use in dialysis-associated anemia or anemia associated with chronic kidney disease has yet been issued. No clear guidelines have been published recommending parameters for initiating IV Fe in oncology. The NCCN and American Society of Hematology/American Society of Clinical Oncology recommend using IV Fe when iron supplementation is indicated.

This article summarizes numerous recent publications showing the efficacy and safety of IV Fe alone and as a component of ESA therapy and proposes a new treatment paradigm incorporating its routine use for treating anemia in oncology patients.

\section{Currently Available Iron Preparations}

Four agents are licensed for use in the United States (Table 1). The first 2 approved were low molecular weight iron dextran (LMW ID) and high molecular weight iron dextran (HMW ID). Both iron dextrans can be given as intravenous boluses in doses up to $400 \mathrm{mg}$ or as a single infusion of the total calculated iron deficit. Reported safety differences in these compounds are discussed in detail later.

The third and fourth agents approved were ferric gluconate and iron sucrose. These agents can be given as short intravenous infusions at doses up to $200 \mathrm{mg}$ for ferric gluconate and $300 \mathrm{mg}$ for iron sucrose, but higher doses have been proscribed. ${ }^{7}$ Both dextrans are approved for use in nephrology and oncology patients, whereas the 2 iron salts are only approved for nephrology patients.

\section{Why IV Fe Works}

Anemia in patients with cancer is believed to reflect a reduction of red cell production by the bone marrow and the interplay of several factors, including a relative decrease in erythropoietin, impaired release of iron from macrophages in the reticuloendothelial system, and diminished or hyporesistance to endogenous erythropoietin by erythroid precursors in the marrow.

In a large subset of patients with cancer-related anemia, an important response-limiting factor is the presence of functional iron deficiency (FID), characterized by an imbalance between iron needs in the erythropoietic marrow and iron supply, which depends on adequacy of iron stores and its rate of mobilization. When supraphysiologic doses of erythropoietin are administered, red cells are produced at a rate that outstrips labile iron availability, causing FID. FID is characterized by hypoferremia, normal or decreased transferrin, and a low transferrin saturation (TSAT) in the presence of adequate storage iron. FID may account for the lack of response in patients who do not experience an adequate response to ESAs.

\begin{tabular}{|c|c|c|c|c|}
\hline & LMW ID & Iron Saccharate & Ferric Gluconate & HMW ID \\
\hline Test dose required & Yes & No & No & Yes \\
\hline Vial volume & $2 \mathrm{~mL}$ & $5 \mathrm{~mL}$ & $5 \mathrm{~mL}$ & $1-2 \mathrm{~mL}$ \\
\hline Mg iron per $\mathrm{mL}$ & $50 \mathrm{mg} / \mathrm{mL}$ & $20 \mathrm{mg} / \mathrm{mL}$ & $12.5 \mathrm{mg} / \mathrm{mL}$ & $50 \mathrm{mg} / \mathrm{mL}$ \\
\hline Black-box warning & Yes & No & No & Yes \\
\hline Total-dose infusion & Yes & No & No & Yes \\
\hline Premedication & TDI only & No & No & TDI only \\
\hline Preservative & None & None & Benzyl alcohol & None \\
\hline $\begin{array}{l}\text { Molecular weight measured by } \\
\text { manufacturer }\end{array}$ & $165,000 \mathrm{Da}$ & $34,000-60,000 \mathrm{Da}$ & $289,000-440,000 \mathrm{Da}$ & $265,000 \mathrm{Da}$ \\
\hline
\end{tabular}

Abbreviation: HMW ID, high molecular weight iron dextran; LMW ID, low molecular weight iron dextran; TDI, total dose infusion. 
Intravenous Iron in Oncology

The distribution of body iron stores underscores the importance of iron in red cell production. Normally, most iron available for erythropoiesis comes from catabolism of red blood cells by macrophages in the reticuloendothelial system. Approximately $70 \%$ of total body iron is found in circulating erythrocytes and approximately $20 \%$ is stored as tissue ferritin, primarily in the liver. Smaller amounts are coupled with enzymes, such as myoglobin. ${ }^{8}$ Iron is absorbed from the duodenum and proximal jejunum. On entering circulation, iron is rapidly transferred to the carrier protein transferrin, which delivers it to all cells in the body. Approximately $80 \%$ of absorbed iron is delivered to the bone marrow.

Erythropoiesis involves close interaction between iron and erythropoietin. Essentially, erythropoietin is the hormone that drives erythropoiesis, providing fuel for the production of red blood cells. When iron and erythropoietin are appropriately coupled, red cell production occurs briskly and efficiently; when one component is deficient, anemia results. Even when both components are present in adequate quantities, they must be coordinately delivered to the bone marrow for proper action.

After the erythropoietin gene was cloned in the 1980s, the product became available for clinical use in 1989. The hematocrit of patients with chronic renal failure rose dramatically when erythropoietin was administered. Enhanced red cell production manifested as a rise in the plasma iron turnover and a decrease in the half-life of iron in the plasma. The plasma ferritin level of patients dropped substantially despite adequate iron stores. This was the first indication that erythropoietin used in high doses could drive red cell production more rapidly than iron could be delivered. ${ }^{9}$

Rutherford et al. ${ }^{8}$ dramatically showed the interplay between iron and erythropoietin. In a study to determine whether erythropoietin could raise baseline hemoglobin to a level that would enhance autologous blood donation, they administered oral iron supplementation during erythropoietin treatment. Baseline hemoglobin levels increased by $1 \mathrm{~g} / \mathrm{dL}$, reflecting enhanced erythropoiesis. Subjects were unable to maintain adequate serum iron levels despite oral iron supplementation. TSAT at onset was $40 \%$, but decreased to $10 \%$ within 2 weeks of erythropoietin treatment. These results showed that developing red blood cells removed iron from circulating transferrin at a rate faster than it could be replaced by either the iron stores or orally absorbed iron.

Hepcidin has emerged as a primary regulator of iron homeostasis. It is a 25-amino acid polypeptide produced by hepatocytes that is an acute-phase protein and the major hormone regulator of iron balance. It inhibits iron release from macrophages in storage sites, diminishing its recycling, and plays a major role in enteral iron absorption through interaction with the transmembrane exporter, ferroportin, accelerating its degradation and resulting in decreased entrance of iron into circulating blood plasma. ${ }^{10}$ In a substantial number of patients with cancer- and chemotherapy-related anemia, inflammatory cytokines are released, causing upregulation of hepcidin with resulting hypoferremia. In these clinical settings, oral administration is inadequate. However, when inflammatory cytokines are not present, oral iron absorption occurs more freely.

Although ESAs have provided a safe and effective option for treating cancer-and chemotherapy-related anemia, not all patients experience adequate response. A lack of available labile iron is the most frequently encountered cause of suboptimal response to ESAs, and unbalanced iron metabolism is a major factor. Control of iron balance requires coordination among sites of uptake, use, and storage. The hepcidin-ferroportin interaction is critical for normal iron homeostasis.

In patients with chronic renal failure, FID is the most common cause of inadequate response to ESAs. Studies in renal failure have shown that IV Fe can correct FID more effectively than oral iron and is now standard care. An understanding of FID and the importance of maintaining an adequate supply of labile iron to meet the needs of the ESA-stimulated erythroid precursors is fundamental. Oral formulations of iron have been shown to be suboptimal and therefore cannot provide labile iron rapidly enough to supply the accelerated erythropoiesis that occurs when ESAs are administered. In contrast, IV Fe provides sufficient quantities at a rate that optimizes efficacy.

\section{Studies of IV Fe in Oncology}

The first study to show the benefit of IV Fe in oncology was published in $2004 .{ }^{11}$ In this study, 155 patients with chemotherapy-related anemia received erythropoietin, 40,000 U subcutaneously per week and either no iron; ferrous sulfate, $325 \mathrm{mg}$ twice daily; 100 -mg boluses of iron dextran weekly to the calculated total dose; 
Auerbach and Ballard

or a single total dose infusion of iron dextran given over 4 hours. Patients randomized to total dose infusion received $125 \mathrm{mg}$ of methylprednisolone pre- and postinfusion to prevent the arthralgias and myalgias ${ }^{12}$ associated with this method of IV Fe administration. ${ }^{13} \mathrm{No}$ differences were seen in baseline serum ferritin levels $(294,290,207$, and $240 \mathrm{ng} / \mathrm{mL}$, respectively) or TSATs (15\%, 18\%, 19\%, and 14\%, respectively) among the 4 groups.

Although no differences in response rates were observed between the IV Fe arms, statistically significant improvements in hemoglobin and hematopoietic responses occurred in individuals who received IV Fe compared with those who did not. The time to reach the target hemoglobin was $60 \%$ shorter in the IV Fe groups, and the total ESA exposure was lower.

In this study, 81 patients received IV Fe, 79 received LMW ID, and 2 received HWM ID when LMW ID was unavailable for a short time. One individual who received HMW ID experienced anaphylactic shock requiring intubation. Months later, off-study, this patient received LMW ID uneventfully. Entry criteria included a serum ferritin of $200 \mathrm{ng} / \mathrm{mL}$ or less or $300 \mathrm{ng} / \mathrm{mL}$ or less, and a TSAT of less than $19 \%$. Although the mean serum ferritin was greater than $200 \mathrm{ng} / \mathrm{mL}$ and the responses were independent of baseline ferritins and TSAT, this study has been criticized for treating mainly patients with iron deficiency.

Recently, reports of 4 clinical trials corroborating the results of this earlier study were published. In the first, Henry et al..$^{14}$ published results of 187 patients with chemotherapy-related anemia and serum ferritins between 100 and $900 \mathrm{ng} / \mathrm{mL}$ or TSAT between $15 \%$ and $35 \%$ who were scheduled to be treated with epoetin, 40,000 U subcutaneously per week. The investigators randomized these patients to treatment with 8 weeks of $125 \mathrm{mg}$ of intravenous ferric gluconate weekly, $325 \mathrm{mg}$ of oral ferrous sulfate 3 times daily, or no iron. No differences were seen in baseline serum ferritin levels (321.5, 373.9, and $388.2 \mathrm{ng} / \mathrm{mL}$, respectively) or TSATs $(29.4 \%, 29.1 \%$, and $36.3 \%$, respectively) among the 3 groups, although statistically significantly greater hemoglobin and hematopoietic responses were seen in the group receiving IV Fe.

Although patients with TSAT less than $20 \%$ receiving IV Fe showed a greater magnitude of hemoglobin rise and response rates, significantly greater hemoglobin and hematopoietic responses were seen in patients with TSAT greater than 20\% compared with those receiving oral ferrous sulfate or no iron. A recent reanalysis of the data, presented at the 2007 American Society of Hematology meeting, showed a significant decrease in thromboembolic events in the ferric gluconate arm compared with the epoietin alone arm. ${ }^{15}$ No significant toxicity attributable to ferric gluconate was seen.

In the second corroborating trial, Hedenus et al. ${ }^{16}$ performed a multicenter randomized trial in 60 patients with lymphoproliferative malignancies and anemia not on chemotherapy. This study was unique in its entry requirements of serum ferritin less than $800 \mathrm{ng} / \mathrm{mL}$ and positive stain for bone marrow hemosiderin before randomization. The mean serum ferritin among the groups was $128 \mathrm{ng} / \mathrm{mL}$ (range, $22-794 \mathrm{ng} / \mathrm{mL}$ ) and the mean TSAT was $21.5 \%$ (range, $5 \%-45 \%$ ). Patients were then randomized into 2 treatment groups receiving epoietin beta 30,000 U subcutaneously per week alone or with iron sucrose administered as $100 \mathrm{mg}$ intravenously on alternate weeks from weeks 8 to 14 .

Significantly greater increases in mean hemoglobin levels were seen from week 8 onward in the iron group. The number of patients experiencing at least a 2-g increment in hemoglobin was nearly doubled in the iron group (53\% vs. 93\%; $P=.001$ ). Despite more missed doses in the no-iron group, the mean weekly epoietin dose after 15 weeks of therapy was $25 \%$ lower in the iron group. Using Swedish pharmacy pricing at the time of the study, the decrement in ESA dosing translated to a savings of $\$ 100$ per patient per week. No significant toxicity was seen in the patients receiving iron sucrose.

In the third trial, Bastit et al. ${ }^{17}$ studied 396 patients with nonmyeloid malignancies and CIA. Unlike the prior 2 studies, patients in this study received darbepoietin, $500 \mathrm{mcg}$, subcutaneously every 3 weeks alone or with intravenous iron sucrose or ferric gluconate weekly for 16 weeks. No differences were seen in baseline serum ferritin levels (279.9 and 278.9 $\mathrm{ng} / \mathrm{mL})$ or TSAT $(21.2 \%$ and $23.7 \%)$ between the groups. Only 4 patients in both groups were truly iron deficient, defined as having a TSAT less than 20\% and serum ferritin less than $15 \mathrm{ng} / \mathrm{mL}$. This study corroborated the results of previous trials, with significantly higher hemoglobin and hematopoietic responses in the IV Fe group. However, unlike the other studies, this study showed a significantly decreased number of transfusions in the IV Fe group compared with the no iron group (Figure 1). ${ }^{18}$ No significant toxicities were seen with IV Fe. 
Intravenous Iron in Oncology

In the fourth trial, Pedrazzoli et al. ${ }^{19}$ studied 149 patients with CIA who received either $150 \mathrm{mcg}$ of darbepoetin subcutaneously per week alone, or $125 \mathrm{mg}$ of ferric gluconate intravenously for the first 6 weeks. The darbepoetin dose was doubled one time to $300 \mathrm{mcg} / \mathrm{wk}$ if a $1-\mathrm{g} / \mathrm{dL}$ increment in hemoglobin was not attained in 4 weeks.

This trial was unique because it excluded patients with absolute or functional iron deficiency by requiring a serum ferritin level of $100 \mathrm{ng} / \mathrm{mL}$ or more and a TSAT of $20 \%$ or more. Exclusion criteria were serum ferritins greater than $800 \mathrm{ng} / \mathrm{mL}$ and a TSAT greater than $40 \%$. No differences were seen in baseline serum ferritin levels ( 350.7 and $333 \mathrm{ng} / \mathrm{mL}$ ) or TSATs $(30.6 \%$ and $27.6 \%)$ between the groups. The IV Fe group had statistically significantly more hematopoietic responses compared with the no-iron group $(92.5 \%$ vs. $70 \%)$. No significant toxicity was associated with ferric gluconate. These results were also corroborated by a study presented by Bellet et al. ${ }^{19}$ at the 2006 American Society of Clinical Oncology Meeting.

These 5 trials included 920 patients; all showed significant improvement in hemoglobin and hematopoietic responses in patients randomized to receive IV Fe compared with oral iron or none. One study showed a statistically significantly lowered number of transfusions. ${ }^{17}$ All responses to IV Fe in the CIA trials were independent of baseline iron parameters. No significant toxicity related to IV Fe was seen in any of the

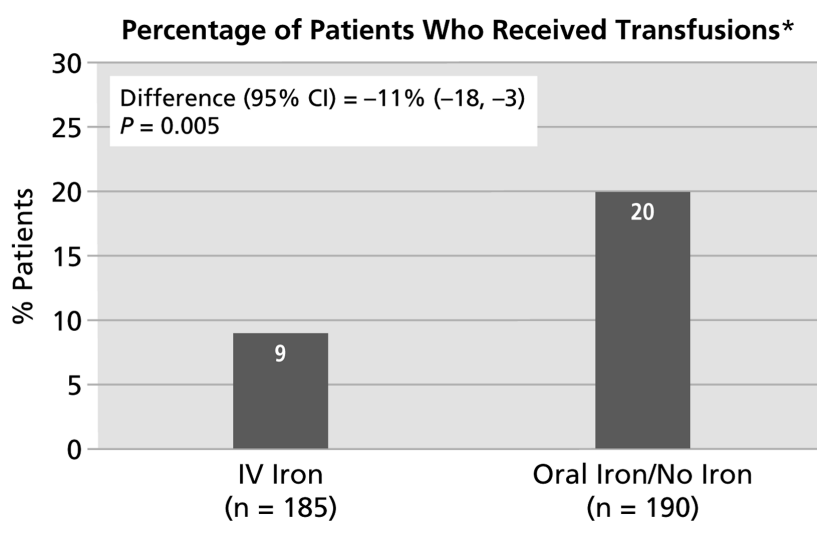

Figure 1 Percentage of patients who received transfusions. *For patients enrolled in the study for at least 29 days Abbreviations: CI, confidence interval; IV, intravenous. Source: Bastit L, Vandebroek A, Altintas S. A randomized, multicenter, controlled trial comparing the efficacy and safety of darbepoetin alfa administered every 3 weeks with or without intravenous iron in patients with chemotherapy-induced anemia. J Clin Oncol 2008;26:1616; with permission. studies, except for 1 of 2 patients who received HMW ID when LMW ID was unavailable. The studies with concomitant ESA therapy all showed significantly decreased exposure to ESAs and considerable cost savings. Results of a recently completed trial comparing 5 treatments of either 300 or 500 mcg of subcutaneous darbepoetin every 3 weeks alone or with $400 \mathrm{mg}$ of LMW ID administered over 30 to 60 minutes are eagerly awaited.

\section{Toxicities of Currently Available Iron Preparations}

The most serious toxicity associated with IV Fe is anaphylactic shock. The authors believe that fear of anaphylaxis is why IV Fe is underused, despite 5 recent publications showing its safety and efficacy in synergizing with ESAs. The overwhelming number of anaphylactic reactions reported are caused by the HMW ID products Imferon (Fisons Pharmaceuticals, Homes Chapel, England), which is no longer available, and Dexferrum (American Regent Labs, Shirley, NY), which is not recommended. ${ }^{21-24}$

The first paper to indicate this was published by Chertow et al..$^{25}$ in 2004 and updated in $2006 .{ }^{26}$ This study, a retrospective analysis of 50 million doses of IV Fe based on adverse events reported to regulatory agencies, showed that when severe adverse reactions were stratified by iron product, virtually all were caused by HMW ID, with an estimated incidence of serious adverse events less than 1:200,000 with LMW ID, iron sucrose, and ferric gluconate.

Unfortunately, a general lack of knowledge about the currently available preparations exists outside the nephrology community. Hospital pharmacists may purchase the less-expensive HMW ID as a generic equivalent, despite different J-codes assigned by the FDA in 2005 and numerous publications showing a drastically higher serious adverse event rate with HMW ID.

Not all reactions to IV Fe are severe; LMW ID, iron sucrose, and ferric gluconate may be associated with minor, self-limiting, infusion-related reactions. Chest and back pain may occur without hypotension, tachypnea, tachycardia, wheezing, stridor, or periorbital edema in approximately 1 of 200 patients receiving LMW ID. This reaction subsides without treatment and does not occur with rechallenge. ${ }^{27}$

However, medical personnel unfamiliar with this reaction can often administer diphenhydramine or 
Auerbach and Ballard

epinephrine inappropriately, which cause significant vasoactive reactions that may mimic anaphylaxis and be incorrectly attributed to the iron preparation. Premedication with diphenhydramine before the IV Fe test dose, which may cause the same misinterpretation of adverse events, has been shown to be ineffective ${ }^{13}$ and therefore should be abandoned. ${ }^{27}$ Premedication is only appropriate using methylprednisolone before and after total dose infusion of LMW ID. ${ }^{12}$ This premedication has been shown to ameliorate the arthralgias and myalgias that occur frequently with this method of IV Fe administration.

Ferric gluconate and iron sucrose can be safely administered as bolus injections or short intravenous infusions in doses up to $300 \mathrm{mg}$. However, higher doses are associated with frequent, annoying, dosedependent gastrointestinal and vasoactive reactions in up to $30 \%$ of patients, and therefore should be avoided. ${ }^{7}$ Based on numerous publications in large series of nephrology and oncology patients, when HMW ID is avoided, IV Fe can be administered with minimal toxicity.

Until 2007, no prospective comparison study of LMW ID, iron sucrose, or ferric gluconate had been performed. Monheim and Bhandari ${ }^{28}$ compared 144 patients undergoing hemodialysis receiving LMW ID with 110 receiving iron sucrose. Patients were treated with one agent for 6 months and then crossed over to the other for an additional 6 months. No anaphylaxis was seen in either group. The 2 forms of IV Fe were equally effective and safe. The use of LMW ID resulted in a savings of $£ 77$ per patient per 6 -month period.

In a study of 60 patients equally divided between LMW ID and iron sucrose, Sav et al..$^{29}$ saw no serious reactions in either group and showed that the 2 therapies were equally effective and safe. These data were confirmed by a systematic review of 60 studies. ${ }^{30}$ Longterm toxicity of IV Fe may be a concern; however, in 14 years of experience with the routine use of IV Fe in nephrology, Kalantar-Zadeh et al. ${ }^{31}$ showed none associated with serum ferritins up to $1200 \mathrm{ng} / \mathrm{mL}$ and TSATs of $40 \%$.

\section{Economic Analysis of IV Fe}

Approximately 380,000 patients are currently receiving ESAs for CIA. The cost of 1 complete course of IV Fe adds little to the cost of ESA therapy. A recent cost analysis in 60 patients in a community oncology prac- tice that routinely administers IV Fe to patients receiving ESAs for CIA was presented at the 2007 meeting of the American College of Clinical Pharmacy. ${ }^{32}$ The cost analysis for ESA and ESA plus iron included office visits, administration expenses, and pharmacy and drug costs. The cost of ESA therapy plus IV Fe was compared with the average cost of ESA therapy in the published norms for patients with CIA. The addition of IV Fe resulted in a cost savings of $\$ 1300$ per patient. However, in the subset of patients with non-small cell lung cancer, the addition of IV Fe saved $\$ 5300$ per patient (Figure 2). Overall, this translates to a cost savings of $\$ 100$ per patient per week, which is consistent with the data from Hedenus et al. ${ }^{16}$ based on the value of the Norwegian krona at the end of 2006.

\section{Conclusions}

In all published studies, IV Fe significantly improved the hemoglobin and hematopoietic responses of patients with CIA receiving ESAs, without substantially increasing toxicity. In approximately 1000 patients receiving thousands of doses of IV Fe, only 1 serious adverse event was recorded, and this event occurred in 1 of 2 patients who received HMW ID. In every study, IV Fe decreased ESA exposure and time to reach target hemoglobin. Large cost savings are realized with routine use of IV Fe with ESAs. A trend is occurring toward a decreased transfusion requirement with IV Fe. ${ }^{17}$ In all studies, the results were independent of the

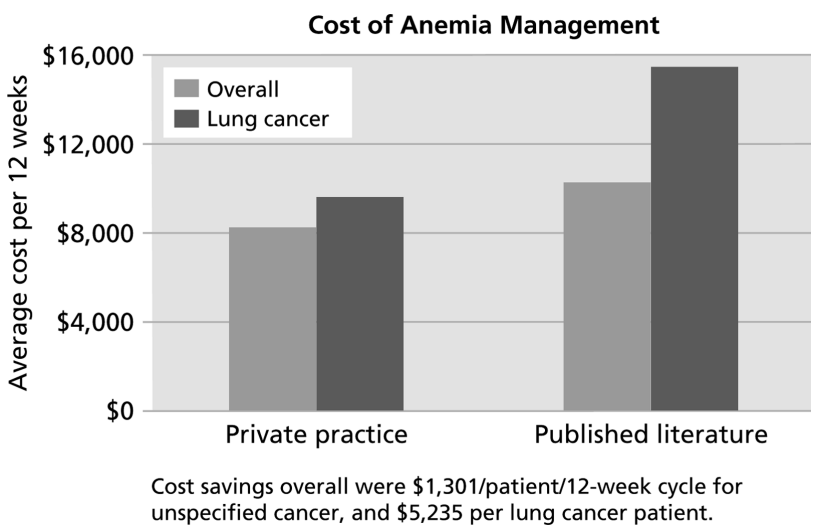

Figure 2 Cost of anemia management. Cost savings overall were $\$ 1301$ per 12-week cycle for unspecified cancer and $\$ 5235$ per patient with lung cancer.

Source: Auerbach M, Pappadakis J, Doherty E. Therapeutic and financial optimization of anemia management in cancer patients with chemotherapy-related anemia through low molecular weight (LMW) iron dextran administration. Presented at the Annual Meeting of the American College of Clinical Pharmacy; Denver, Colorado; October 14-17, 2007. Abstract 188 
Intravenous Iron in Oncology

baseline iron parameters serum ferritin and TSAT. In oncology, the safety data exists up to ferritins of $1000 \mathrm{ng} / \mathrm{mL}$ and TSATs of $40 \%$.

Considering the great improvement in efficacy and cost without increased toxicity, the authors believe sufficient data recommend the use of IV Fe in patients receiving ESAs for CIA who are unresponsive after 4 weeks. For patients receiving frequent doses of ESAs during chemotherapy, LMW ID, iron sucrose, and ferric gluconate can be given over short periods in doses up to $300 \mathrm{mg}$ with equal safety and efficacy. For total dose infusion, only LMW ID should be used. For patients with absolute iron deficiency, full IV Fe replacement should be given before ESAs. Currently, IV Fe is underused in oncology.

\section{References}

1. National Institute for Health and Clinical Excellence. Anaemia (cancer-treatment induced) - erythropoietin (alpha and beta) and darbepoetin: appraisal consultation. Available at: http://www. $\underline{\text { nice.org.uk/guidance/index.jsp?action }=\text { article } \& r=\text { true } \& o=37607}$. Accessed May 27, 2008.

2. Henke M, Laszig R, Rube C, et al. Erythropoietin to treat head and neck cancer patients with anaemia undergoing radiotherapy: randomised, double-blind, placebo-controlled trial. Lancet 2003;362: 1255-1260.

3. Leyland-Jones B, Semiglazov V, Pawlicki M, et al. Maintaining normal hemoglobin levels with epoetin alfa in mainly nonanemic patients with metastatic breast cancer receiving first-line chemotherapy: a survival study. J Clin Oncol 2005;23:5960-5972.

4. Wright JR, Ung YC, Julian JA, et al. Randomized, double-blind, placebo-controlled trial of erythropoietin in non-small-cell lung cancer with disease-related anemia. J Clin Oncol 2007;25:1027-1032.

5. Glaspy JA. The development of erythropoietic agents in oncology. Expert Opin Emerg Drugs 2005;10:553-567.

6. Collins A. Trend in iron testing and IV iron dosing in cancer patients receiving chemotherapy and ESA versus dialysis patients. Presented at the American Society of Hematology 45th Annual Meeting and Exposition; San Diego, California; December 6-9, 2003.

7. Chandler G, Harchowal J, Macdougall IC. Intravenous iron sucrose: establishing a safe dose. Am J Kidney Dis 2001;38:988-991.

8. Rutherford CJ, Schneider TJ, Dempsey H, et al. Efficacy of different dosing regimens for recombinant human erythropoietin in a simulated perisurgical setting: the importance of iron availability in optimizing response. Am J Med 1994;96:139-145.

9. Hotta $\mathrm{T}$, Ogawa $\mathrm{H}$, Saito A, Ito A. Iron balance following recombinant human erythropoietin therapy for anemia associated with chronic renal failure. Int J Hematol 1991;54:195-200.

10. Ganz T. Hepcidin, a key regulator of iron metabolism and mediator of anemia of inflammation. Blood 2003;102:783-788.

11. Auerbach $M$, Ballard $H$, Trout JR, et al. Intravenous iron optimizes the response to recombinant human erythropoietin in cancer patients with chemotherapy-related anemia: a multicenter, openlabel, randomized trial. J Clin Oncol 2004;22:1301-1307.

12. Auerbach $M$, Chaudhry M, Goldman H, Ballard H. Value of methylprednisolone in prevention of the arthralgia-myalgia syndrome associated with the total dose infusion of iron dextran: a double blind randomized trial. J Lab Clin Med 1998;131:257-260.

13. Auerbach $M, W i t t D$, Toler W, et al. Clinical use of the total dose intravenous infusion of iron dextran. J Lab Clin Med 1988;111:566-570.

14. Henry DH, Dahl NV, Auerbach $\mathrm{M}$, et al. Intravenous ferric gluconate significantly improves response to epoetin alfa versus oral iron or no iron in anemic patients with cancer receiving chemotherapy. Oncologist 2007;12:231-242.

15. Henry DH, Dahl NV, Auerbach M. Is thromboembolism in cancer patients treated with erythropoietic stimulating agents related to thrombocytosis and iron restricted erythropoiesis [abstract]? Blood 2007;110:Abstract 1625.

16. Hedenus M, Birgegard $G$, Nasman $P$, et al. Addition of intravenous iron to epoetin beta increases hemoglobin response and decreases epoetin dose requirement in anemic patients with lymphoproliferative malignancies: a randomized multicenter study. Leukemia 2007;21:627-632.

17. Bastit L, Vandebroek A, Altintas S. A randomized, multicenter, controlled trial comparing the efficacy and safety of darbepoetin alfa administered every 3 weeks with or without intravenous iron in patients with chemotherapy-induced anemia. J Clin Oncol 2008;26: $1611-1618$.

18. Pinter T, Mossman T, Suto J, Vansteenkiste J. Effects of intravenous (IV) iron supplementation on responses to every-3-week (Q3W) darbepoetin alfa (DA) by baseline hemoglobin in patients (pts) with chemotherapy-induced anemia (CIA) [abstract]. J Clin Oncol 2007;25(Suppl 1):519s. Abstract 9106.

19. Pedrazzoli P, Farris A, Del Prete S, et al. Randomized trial of intravenous iron supplementation in patients with chemotherapy related anemia without iron deficiency treated with darbepoetin alfa. J Clin Oncol 2008;26:1619-1625.

20. Bellet RE, Ghazal H, Flam M, et al. A phase 3 randomized controlled study comparing iron sucrose intravenously to no iron treatment of anemia in cancer patients undergoing chemotherapy and erythropoietin stimulating agent therapy [abstract]. J Clin Oncol 2007;25(Suppl 1):519s. Abstract 9109.

21. Case G. Maintaining iron balance with total-dose infusion of intravenous iron dextran. ANNA J 1998; 25:65-68.

22. Fletes R, Lazarus JM, Gage J, Chertow GM. Suspected iron dextranrelated adverse drug events in hemodialysis patients. Am J Kidney Dis 2001;37:743-749.

23. Mamula P, Piccoli DA, Peck SN, et al. Total dose intravenous infusion of iron dextran for iron-deficiency anemia in children with inflammatory bowel disease. J Pediatr Gastroenterol Nutr 2002; 34:286-290.

24. McCarthy JT, Regnier CE, Loebertmann CL, Bergstralh EJ. Adverse events in chronic hemodialysis patients receiving intravenous iron dextran-a comparison of two products. Am J Nephrol 2000;20:455-462.

25. Chertow GM, Mason PD, Vaage-Nilsen O, Ahlmen J. On the relative safety of parenteral iron formulations. Nephrol Dial Transplant 2004;19:1571-1575.

26. Chertow GM, Mason PD, Vaage-Nilsen O, Ahlmen J. Update on adverse drug events associated with parenteral iron. Nephrol Dial Transplant 2006;21:378-382.

27. Auerbach M, Ballard $\mathrm{H}$, Glaspy J. Clinical update: intravenous iron for anaemia. Lancet 2007;369:1502-1504.

28. Monheim K, Bhandari S. Tolerability and efficacy of parenteral iron therapy in hemodialysis patients, a comparison of preparations. Transfusion Alternatives in Transfusion Medicine 2007;9:37-2.

29. Sav T, Tokgoz B, Sipahioglu MH et al. Is there a difference between the allergic potencies of the iron sucrose and low molecular weight iron dextran? Ren Fail 2007;29:423-426. 
Auerbach and Ballard

30. Critchley J, Dundar Y. Adverse events associated with intravenous iron infusion (low-molecular-weight iron dextran and iron sucrose): a systematic review. Transfusion Alternatives in Transfusion Medicine 2007;9:8-36.

31. Kalantar-Zadeh K, Regidor DL, McAllister CJ, et al. Time-dependent associations between iron and mortality in hemodialysis patients. J Am Soc Nephrol 2005;16:3070-3080.

\section{Iron Replacement in Oncology}

To obtain credit, you should first read the journal article. After reading the article, you should be able to answer the following, related, multiple-choice questions. To complete the questions and earn continuing medical education $(\mathrm{CME})$ credit, please go to http://www.medscape.com/cme/jnccn.

Credit cannot be obtained for tests completed on paper, although you may use the worksheet below to keep a record of your answers. You must be a registered user on Medscape.com. If you are not registered on Medscape. com, please click on the New Users: Free Registration link on the left hand side of the website to register.

Only one answer is correct for each question. Once you successfully answer all post-test questions you will be able to view and/or print your certificate. For questions regarding the content of this activity, contact
32. Auerbach M, Pappadakis J, Doherty E. Therapeutic and financial optimization of anemia management in cancer patients with chemotherapy-related anemia through low molecular weight (LMW) iron dextran administration. Presented at the Annual Meeting of the American College of Clinical Pharmacy; Denver, Colorado; October 14-17, 2007. Abstract 188.

the accredited provider, CME@medscape.net. For technical assistance, contact CME@webmd.net.

American Medical Association's Physician's Recognition Award (AMA PRA) credits are accepted in the US as evidence of participation in CME activities. For further information on this award, please refer to http://www.ama-assn.org/ama/pub/category/ 2922.html. The AMA has determined that physicians not licensed in the US who participate in this CME activity are eligible for AMA PRA Category 1 Credits $^{\mathrm{TM}}$. Through agreements that the AMA has made with agencies in some countries, AMA PRA credit is acceptable as evidence of participation in CME activities. If you are not licensed in the U.S. and want to obtain an AMA PRA CME credit, please complete the questions online, print the certificate and present it to your national medical association.
1. Which of the following agents is most likely to be approved for use in both oncology and nephrology patients with anemia?

A. Low-molecular-weight dextran

B. Iron sucrose

C. Ferric gluconate

D. None of the above

2. Anemia occurring in oncology patients is most likely to reflect which of the following processes?

A. Decrease in erythropoietin

B. Impaired release of iron from macrophages

C. Hyporesistance to endogenous erythropoietin by erythroid precursors

D. All of the above

3. Which of the following is least likely to be a characteristic of functional iron deficiency (FID)?

A. Hyperferremia

B. Normal transferrin

\section{Activity Evaluation}

1. The activity supported the learning objectives.
Strongly Disagree
Strongly Agree

123

$4 \quad 5$

2. The material was organized clearly for learning to occur.

Strongly Disagree

$\begin{array}{lll}1 & 2 & 3\end{array}$

Strongly Agree

4
C. Low transferrin saturation
D. Adequate storage iron

4. Which of the following is the most frequently encountered cause of suboptimal response to ESAs in the treatment of cancer- and chemotherapy-related anemia in the absence of chronic renal failure?
A. Lack of available labile iron
B. Interaction with other therapies
C. Presence of metastases
D. None of the above

5. All trials of intravenous iron in patients with cancerinduced anemia have shown which of the following effects?
A. Improved mortality
B. Improved hemoglobin after ESA use
C. No effect on hemoglobin
D. Anaphylaxis as a common adverse effect

To obtain credit, go to Medscape online at: http://www.medscape.com/cme/jnccn.

3. The content learned from this activity will impact my practice.

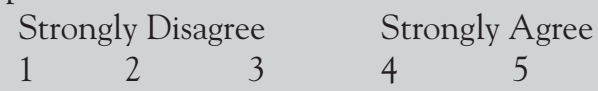

4. The activity was presented objectively and free of commercial bias.

Strongly Disagree Strongly Agree

$\begin{array}{lllll}1 & 2 & 3 & 4 & 5\end{array}$ 